\title{
Reflexiones en torno a la participación en el desarrollo rural. ¿Reparto social o reforzamiento del poder? LEADER $y$ PRODER en el sur de Espańa
}

Francisco Navarro. Universidad de Granada, Granada, España.

Eugenio Cejudo. Universidad de Granada, Granada, España.

Juan Carlos Maroto. Universidad de Granada, Granada, España.

RESUMEN | La participación es uno de los pilares esenciales del desarrollo rural. Ahora bien, diversas causas (control político, predominio del control descendente, excesivo enfoque en los resultados económicos, etcétera) han provocado que los beneficios principales de los Programas de Desarrollo Rural (PDR) hayan quedado en manos de unos pocos, las elites económicas y políticas locales. Los colectivos desfavorecidos (mujeres, jóvenes, trabajadores del campo, desempleados, etcétera) han sido excluidos. De lo anterior se deriva que la práctica del desarrollo rural origina un reforzamiento del poder. Esto se comprueba mediante el análisis de la aplicación de los PDR en la provincia de Granada para el periodo 1991-2006.

PALABRAS CLAVE | desarrollo regional y local, gobierno local, planificación del desarrollo.

ABSTRACT | Participation is a basic pillar of rural development. However, for several reasons (ie. political control, predominant top-down control models, focus on economic projects, etc.), the main contributions of Rural Development Programmes (RDP's) are controlled by a limited group of people - mainly local economic and political elites. Disadvantaged groups (such as women, young people, farm workers, unemployed, etc.) have been excluded. As such, it follows that rural development applications have a tendency to reinforce existing power structures. This is corroborated by an analysis of the implementation of RDP's in the province of Grenade, from 1991 to 2006.

KEY WORDS | regional and local development, local government, development planning.

Recibido el 12 de octubre de 2012, aprobado el 21 de noviembre de 2013

Email: Francisco Navarro, favalver@ugr.es | Eugenio Cejudo, cejudo@ugr.es | Juan Carlos Maroto, jcmaroto@ugr.es 


\section{Introducción: hipótesis y estructura del trabajo}

Con la aplicación en los espacios rurales de la Unión Europea (UE) de los Programas de Desarrollo Rural (PDR) -en concreto, de la Iniciativa Comunitaria LEADER (I, II y +) 1, junto con PRODER2 (I y II), entre 1991 y 2006-, se han perseguido varios objetivos, siendo esenciales los siguientes: un desarrollo rural integrado, una diversificación de las economías y un desarrollo ascendente, todo ello promoviendo la participación, el autogobierno y el partenariado. En este artículo nos centraremos en el estudio de este último aspecto, esencial a la hora de promover un desarrollo sostenible y, más concretamente, si se trata de analizar a los beneficiarios finales de tales iniciativas. Nuestro propósito es dar respuesta a dos cuestiones principales: (i) quiénes han sido los beneficiarios; y (ii) las ayudas provenientes de tales programas, ¿han sido controladas por las elites económicas y políticas locales? Ambas cuestiones en el marco de nuestro convencimiento de que, a la hora de determinar el éxito o fracaso de una iniciativa, tiene más importancia analizar cuestiones como quiénes han sido realmente los implicados en los procesos de desarrollo, o si se ha avanzado en la inclusión social de los actores con menos poder y menos oportunidades, que estudiar los propios resultados de los PDR.

Se parte de la siguiente hipótesis: la participación local, promovida tanto por LEADER COmo por PRODER, pese a la gran cantidad de potencialidades que presenta, conlleva también una serie de riesgos, al desplazar la responsabilidad por el desarrollo socioeconómico desde el Estado a las comunidades rurales. Entre tales riesgos está la tendencia a postular que, si no se consiguen avances en ese ámbito, el error es de la comunidad. Por esta vía se generan (o profundizan) desigualdades, surgen "ganadores" y "perdedores", ya que la capacidad de las comunidades locales para participar con éxito en el desarrollo endógeno es desigual. E, incluso, relativamente pocos miembros dentro de una comunidad se implican en la gobernanza local. Usualmente lo hacen quienes poseen tiempo, recursos y aspiraciones para comprometerse; las elites locales, en definitiva, que controlan además otras instituciones y poderes. Por esta vía, otros muchos actores son excluidos del proceso de desarrollo, mientras sus necesidades e intereses se ven postergados; se trata de los más desfavorecidos y débiles: mujeres, jóvenes, trabajadores del campo y desempleados, entre otros. Por tanto, desde nuestro punto de vista, y compartiendo la opinión de Woods, Edwards, Anderson y Gardner (2007), modalidades como las descritas

1 Gracias a la Reforma de los Fondos Estructurales Europeos de 1988, surgieron los Programas de Desarrollo Rural como herramienta para paliar los déficit socioeconómicos de las áreas más deprimidas. Sus principios filosóficos fueron recogidos en el texto comunitario El futuro del mundo rural, y se comenzaron a implantar a partir de 1991 como una iniciativa piloto para estimular enfoques innovadores y participativos en el desarrollo rural a nivel local. Desde 1991 hasta la actualidad, los periodos de programación en los que ha participado han sido: 1991-1993 (LEADER I), 1994-1999 (LEADER II), 2000-2006 (LEADER +), 2007-2013 (se agrupa junto con otras medidas de desarrollo rural en un único instrumento programático denominado Fondo Agrario para el Desarrollo Rural).

2 Se trata de un "LEADER a la española", un programa diseñado por el Estado español para las zonas rurales, con similares objetivos, especificidades y medidas que tal iniciativa; un programa nacional de desarrollo rural diseñado y aplicado en España y cofinanciado por la Unión Europea. 
constituyen nuevas formas de gobernabilidad neoliberales, en las que el papel del Estado se limita a fomentar el espíritu empresarial y contribuir a que las comunidades rurales se ayuden a sí mismas.

Tras exponer los antecedentes y aclarar una serie de conceptos clave en la segunda sección, en la tercera se enmarca y justifica espacial y temporalmente el ámbito de estudio. La cuarta se dedica a presentar el método mixto, cuantitativo y cualitativo, que se ha empleado para abordar la situación en estudio. En la siguiente sección, la quinta, se desenmascara a los beneficiarios finales, se describe quiénes han sido y quiénes no. En la sexta se explican las causas de las ausencias y presencias en la participación final de los PDR. Y, por último, se culmina el artículo con una serie de reflexiones y conclusiones finales.

\section{Participación y poder. Aclaraciones conceptuales y antecedentes}

Con una cierta perspectiva histórica en torno a la aplicación de LEADER en los territorios rurales de la Unión Europea, se puede afirmar que, junto a su inicial visión como un instrumento para abordar los problemas del mundo rural, también fue concebido como un instrumento de poder, y por su control disputaron los diferentes agentes de la escala local (Esparcia, 2011). Ello se refleja en la notable presencia de los gobiernos locales en la composición de los Grupos de Acción Local (GAL); en el entramado de agentes que operan e interactúan a diferentes escalas (de la comunitaria a la local) y que tuvo como primera consecuencia la formación de grupos de presión en el seno de los GAL. En ocasiones adoptaron un carácter sectorial (grupos de agentes relacionados con determinadas actividades: cargos elegidos, representantes culturales, sociales o económicos), y en otras tuvieron un carácter territorial. Los grupos de presión entendieron LEADER como un instrumento para el desarrollo de "su" sector a la vez que de legitimación y/o de poder, que con frecuencia utilizaron de manera partidista (Esparcia, Noguera \& Pitarch, 2000), pese a que se imponía la necesidad de aplicar los principios de la gobernanza territorial en la gestión de las políticas públicas. Este concepto, que surgió en la década de los noventa del siglo pasado, se definió como "el proceso en el que gobernar depende de la colaboración entre un complejo conjunto de instituciones y actores que va más allá del Gobierno" (Stoker, 1998, p. 1), englobando "además de los partidos políticos elegidos, a actores individuales, organizaciones no estatales, empresas, etc." (Ward \& McNicholas, 1998, p. 27). Las comunidades rurales poseen ahora mayor libertad, aunque también mayor responsabilidad, para controlar y gestionar su propio proceso de desarrollo; en definitiva, para tomar decisiones. Esta perspectiva implicaba no solo superar su inicial concepción ligada a la simplificación de la toma de decisiones por parte de los poderes públicos y de la empresa privada en busca de la máxima eficacia económica, sino incluso del "buen gobierno", fundamentada en un papel insustituible del Estado y de un mayor protagonismo de la sociedad civil (Farinós, 2008; Romero \& Farinós, 2011). La gobernanza apunta a la forma de mejorar la relación (horizontal) entre una pluralidad de actores públicos y privados, con el propósito de mejorar la toma de decisiones, la gestión y el desarrollo de lo público y lo colectivo, con una marcada intención de integración y de interdependencia (Jorquera, 2011). 
Necesariamente imbricado en la gobernanza se encuentra el concepto de empoderamiento de los colectivos marginados o escasamente considerados en el mundo rural, y de los que se reclama una participación activa y básica en el resurgir del mundo rural por su implicación, entre otras cuestiones, en los PDR, tanto en el diseño y gestión de dichos programas como en lo que toca a su papel de beneficiarios. Nos referimos, especialmente, a las mujeres y los jóvenes. Por "empoderamiento" entendemos la creación de condiciones objetivas y subjetivas, personales y colectivas, que posibilitan la participación de tales colectivos desfavorecidos en los procesos de toma de decisiones y acceso al poder, así como la toma de conciencia del poder, individual y colectivamente. Los procesos de terciarización del mundo rural, con lo que supone de diversificación de las actividades productivas que está en el origen de la iniciativa LEADER; el nivel formativo alcanzado por estos colectivos de mujeres y jóvenes; el carácter participativo de estos PDR; el importante desarrollo de movimientos asociativos en estos grupos, entre otras cuestiones, contribuyen a generar las condiciones necesarias para lograr el empoderamiento que se pregona y se reclama en los PDR, pero que no se termina de conseguir, por diversos motivos que señalaremos más adelante.

Scott (2004) señala que la gobernanza no constituye un proceso de desarrollo para la población local, sino un proceso de desarrollo con la población local, en el que intervienen todos los colectivos sociales. La participación se convierte en un objetivo del modelo de desarrollo, enfatizándose "la participación en el diseño e implementación de la acción” (Ray, 2001, p. 280). Ateniéndonos a la retórica de la autoayuda, ahora los residentes rurales tienen autonomía y son libres para elegir, y de hecho son llamados - con los PDR- a decidir sobre su propio futuro.

En cuanto a los antecedentes, la hipótesis sobre la desigual participación y distribución de las ayudas de los PDR en las comunidades locales, de las que quedan excluidos los colectivos marginales, es también compartida y demostrada por otra serie de autores: Kearney, Boyle y Walsh (1995), para el caso de Irlanda; Shortall (2008) y Shucksmith (2010) para Escocia; Midmore (1998) para Gales; Ray (2001) en Bretańa; Buller (2000) para Francia; Esparcia et al. (2000) y Lacambra (2001) para Espańa; para Andalucía, Rodríguez (2000) y Moscoso (2005); y otra serie de autores de forma genérica: Scott (2004), Böcher (2008) y Tovey (2008).

\section{Área de estudio y referencia temporal}

Como área de estudio se ha elegido el sur de España; en concreto, los territorios rurales de la provincia de Granada, que son representativos de las problemáticas que afectan a los espacios rurales del sur de la Unión Europea, y de la marginalización de los grupos desfavorecidos en los PDR. Así, en dicha provincia encontramos:

- Un predominio de territorios rurales, que supera el $80 \%$ de la superficie total.

- Una localización periférica tanto en la Unión Europea (UE), como en España. Es decir, una elevada lejanía respecto de los principales centros y nodos difusores de crecimiento económico y centros de decisión principales. 
- Una alta presencia de poblaciones con escaso peso demográfico, predominando los asentamientos que no alcanzan los 5.000 habitantes (montaña Bética andaluza), además de una baja densidad de población, aunque existen algunas pequeñas ciudades que articulan el tejido urbano y que funcionan como ciudades mercado y de servicios, cabeceras comarcales (Guadix, Baza, Loja, etcétera.). Es decir, frente a la decadencia de las áreas rurales más remotas, aparece una concentración poblacional y económica en pequeñas ciudades.

- Un acentuado envejecimiento y pervivencia del éxodo rural, desde la década de los ochenta del siglo pasado hasta la actualidad.

- Un desempleo elevado y estructural, superior al 20\% (Junta de Andalucía, 2008) y que se ha convertido en un círculo vicioso, donde los desempleados "son la mitad del año jornaleros (o mejor la sexta parte del año jornaleros) y la otra mitad subsidiados o el resto subsidiados agrarios" (Ramos \& Romero, 1995, p. 181). El subsidio agrario de desempleo, el denominado actualmente el Plan de Fomento Agrario (PFA) y antiguo Plan de Empleo Rural (PER), es así vital para la supervivencia y mantenimiento de las poblaciones en el ámbito rural.

- Un elevado peso del sector agrario en lo social, territorial, cultural y económico. De hecho, sin embargo, este sector presenta una escasa productividad, debido a la frecuencia de precios bajos en sus productos, explotaciones de reducido tamańo al borde de la viabilidad económica, abundancia de suelos pobres, etcétera. Por tanto, se asiste a una alta dependencia de las ayudas públicas (comunitarias y nacionales) para mantener tanto la producción como el paisaje.

- Derivado del anterior, una renta per cápita y unos estándares de vida considerablemente inferiores a la media comunitaria y nacional, lo que hace a la provincia de Granada receptora de fondos provenientes de la política comunitaria de desarrollo regional; concretamente los dirigidos a las regiones menos desarrolladas de la UE, vale decir, las antiguas regiones de Objetivo 1, hoy denominadas de Convergencia. Tal situación persiste, tal y como recoge el sexto informe de la Comisión de la ue sobre la situación de la cohesión económica y social (Comisión Europea, 2009, p. 295).

- Una alta presencia de la montaña (cordilleras Béticas) y, por ende, de zonas desfavorecidas (más del 74\%).

- Y por último -y como no-, la existencia de una Política Agraria Común y, dentro de ella, de una serie de Programas de Desarrollo Rural que se aplican y despliegan en estos territorios.

Como referencia temporal se ha abarcado el periodo comprendido entre $1991 \mathrm{y}$ 2006. Es decir, los periodos de programación de la UE ya concluidos y, por tanto, para los que se dispone de información definitiva: 1991-1993, 1995-1999 y 20002006. El actual periodo (2007-2013) no termina sino a finales de 2013, y sus resultados definitivos no estarán disponibles hasta fechas bastante posteriores.

Para la puesta en marcha de estos programas se creó, a lo largo de la década de los noventa, una estructura administrativo-territorial en todo el territorio rural 
comunitario, mediante los GAL. En la provincia de Granada el proceso fue idéntico, como no puede ser de otra manera. Surgió en 1992 el primer Gal en las Alpujarras, al que le continuaron para 1995-1999 los de Altiplano, Guadix y Poniente. Más tarde, entre 1996 y 1998, se constituyeron los de Los Montes, Valle de LecrínTemple y Vega-Sierra Elvira. Finalmente, en el período 2000-2006 se configuró un último gal en el Arco Noreste de la Vega. De esta manera, solo 20 municipios de los 168 que integran la provincia quedaron fuera de los $\mathrm{PDR}^{3}$.

FIgURA 1 | Situación y configuración territorial de los Grupos de Acción Local (GAL) granadinos, en los periodos 1991-1993, 1995-1999 y 2000-2006

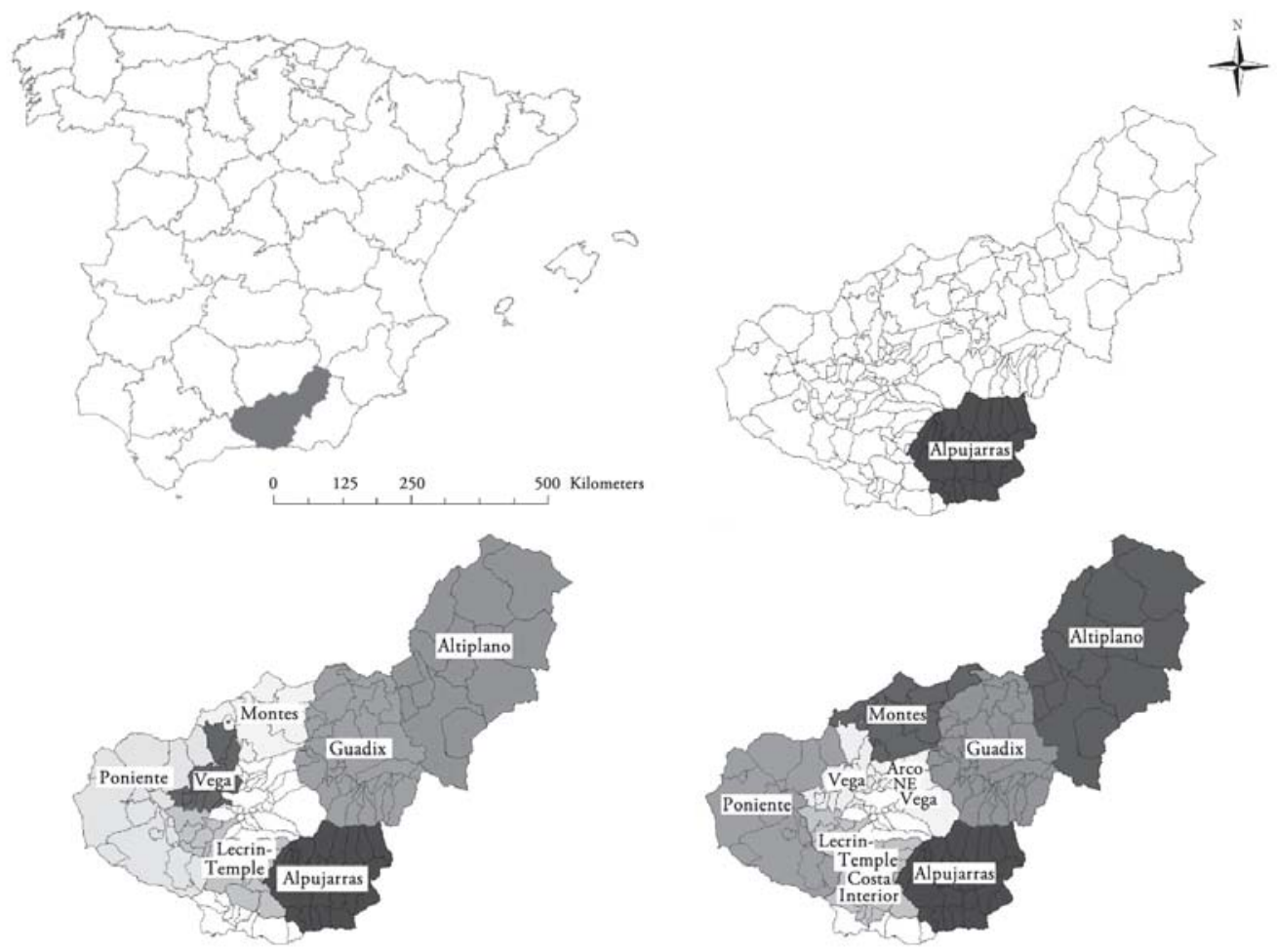

FUENTE ELABORACIÓN PROPIA. DATOS DE LOS INFORMES FINALES DE LOS GAL GRANADINOS

\section{Metodología}

A la hora de afrontar un trabajo de estas dimensiones, y para desentrańar el perfil de los beneficiarios finales de los PDR, se hace necesario combinar las metodologías cuantitativa y cualitativa. Así, se ha trabajado con los datos ofrecidos por la estadística oficial y por los GAL; para estos últimos se ha considerado sus informes finales, donde aparecen diversos indicadores cuantitativos. Por otra parte, se ha recabado información de cada uno de los emprendedores acogidos a los PDR, de los beneficiarios y actores finales, en un cuestionario cumplimentado mediante entrevistas personales (Cuadro 1), con una duración aproximada de 15-20 minutos. Ello nos 
ha permitido dilucidar si los colectivos desfavorecidos de los ámbitos rurales han podido acogerse a las ayudas distribuidas por los PDR.

\section{CUADro 1 | Cuestionario para los emprendedores beneficiados por Programas de Desarrollo Rural (PDR) destinados a los Grupos de Acción Local (GAL) de la provincia de Granada, periodo 1991-2006}

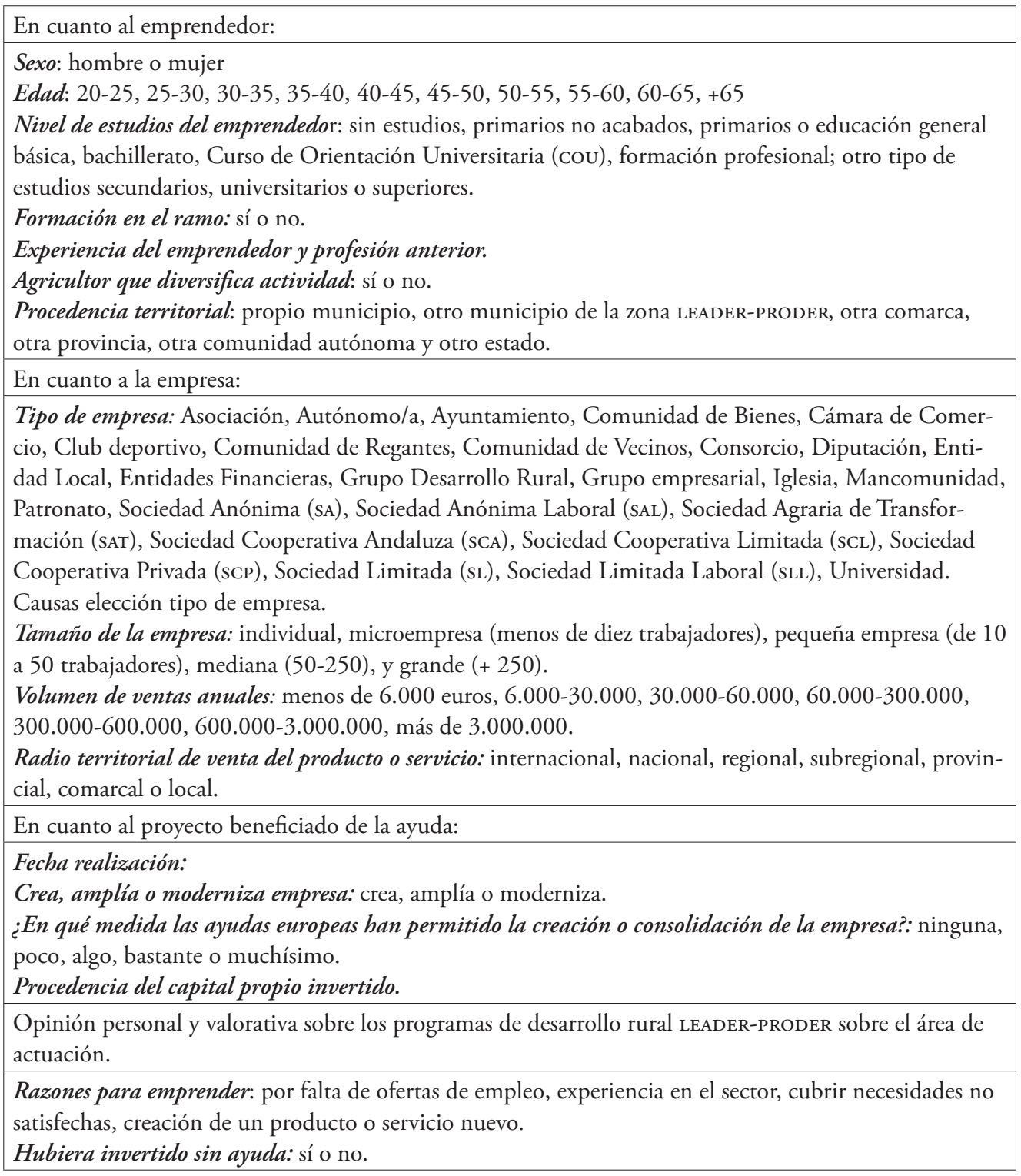

FUENTE ELABORACIÓN PROPIA

Para esta encuesta, de las 2.337 actuaciones en PDR que se llevaron a cabo en los 148 municipios granadinos en el período 1991-2006, se descartaron las entrevistas a promotores públicos, al ser sus inversiones "no productivas", ciñéndonos a las meramente productivas, a las realizadas por emprendedores privados (1.072 proyectos y 949 emprendedores privados). De esta forma se descubriría quiénes fueron 
realmente los participantes finales de los PDR. Se tomó como criterio en la selección que se contemplase la gran variedad existente en la tipología de empresas; que los encuestados formaran parte de las tres medidas productivas (turismo rural; pymes, artesanía y servicios locales; y valorización de la producción agraria, ganadera y forestal); proporcionalidad en el número de hombres y mujeres; $y$, por último, que fueran de diferentes GAL, de distintos periodos de programación y de diferentes municipios, siendo representativos de cada uno de ellos. En total se realizaron 107 entrevistas, lo que supone 11,3\% del universo total (Cuadro 2). Los entrevistados eran los gestores principales de la empresa, es decir, los que se habían implicado en mayor medida tanto en el proyecto solicitado como en la empresa (titulares de la empresa, gerentes de cooperativas); igualmente se recabó información sobre los trabajadores beneficiarios de dichas actuaciones.

CUADro 2 | Esquema de entrevistas realizadas: por Grupo de Acción Local (GAL), sexo, medidas, tipo de empresa

\begin{tabular}{|c|c|c|c|c|c|c|c|c|c|c|c|}
\hline \multirow{2}{*}{ GAL } & \multicolumn{2}{|c|}{ SEXO } & \multicolumn{3}{|c|}{ MEDIDAS } & \multicolumn{5}{|c|}{ TIPO DE EMPRESA } & \multirow{2}{*}{ TOTAL } \\
\hline & $\mathbf{M}$ & $\mathbf{H}$ & III & IV & $\mathbf{v}$ & A & SL & SA & c & $\mathbf{O}$ & \\
\hline A & 3 & 13 & 6 & 4 & 6 & 7 & 5 & 1 & 0 & 3 & 16 \\
\hline B & 2 & 16 & 4 & 7 & 7 & 8 & 4 & 2 & 2 & 2 & 18 \\
\hline $\mathrm{C}$ & 1 & 4 & 2 & 1 & 2 & 1 & 1 & 0 & 2 & 1 & 5 \\
\hline D & 3 & 12 & 5 & 4 & 6 & 7 & 2 & 1 & 2 & 3 & 15 \\
\hline $\mathrm{E}$ & 2 & 9 & 2 & 4 & 5 & 5 & 2 & 1 & 1 & 2 & 11 \\
\hline $\mathrm{F}$ & 5 & 13 & 6 & 6 & 6 & 7 & 3 & 2 & 3 & 4 & 18 \\
\hline G & 5 & 7 & 2 & 7 & 3 & 4 & 2 & 2 & 2 & 2 & 12 \\
\hline $\mathrm{H}$ & 3 & 9 & 2 & 6 & 4 & 4 & 4 & 1 & 1 & 2 & 12 \\
\hline Total & 24 & 83 & 29 & 39 & 39 & 43 & 23 & 10 & 13 & 19 & 107 \\
\hline
\end{tabular}

Gal: A: Alpujarras, B: Altiplano, C: Arco NE Vega, D: Guadix, E: Apromontes, F: Poniente, G: LecrínTemple-CI y H: Vega.

Sexo: H: Mujeres. H: Hombres

Medidas: III. Turismo rural. Iv. Pymes, artesanía y servicios. v. Valorización y comercialización de la producción agraria y endógena.

Tipo de empresa: A: Autónomos. sL: Sociedades Limitadas. SA: Sociedades Anónimas. C: Cooperativas. o: Otros.

FUENTE ELABORACIÓN PROPIA

\section{Los beneficiarios finales}

Las áreas rurales, lógicamente, no están habitadas por grupos homogéneos de personas. Consciente o inconscientemente, las actuaciones planteadas por los PDR, por regla general, no han tenido presente este hecho, y no han obtenido una efectiva participación de todos los colectivos, siendo excluidos los más desfavorecidos: mujeres, jóvenes, desempleados, trabajadores del campo, entre otros; y por tanto, también lo han sido sus necesidades e intereses. 


\section{¿Quiénes no?}

Detengámonos, para comenzar, en los que apenas han aparecido como beneficiarios finales en los PDR. En primer lugar, por su especial importancia y particularidad, nos vamos a referir al colectivo marginal, ya estructural en el medio rural andaluz y, por ende granadino, de los desempleados, uno de los de mayor necesidad de atención por parte de las políticas públicas. Pero los desempleados, salvo determinados cursos de formación, en los que ha existido un enfoque demasiado genérico, sin apenas orientación al mercado profesional, han sido obviados y excluidos del proceso de desarrollo. Incluso, apenas han existido experiencias de autoempleo en las que la participación de los PDR haya sido significativa o haya generado un efecto demostrativo importante ${ }^{4}$.

Otro colectivo, muy relacionado con el anterior por el carácter temporal y escaso del trabajo que realizan, es el de los jornaleros, que tampoco ha participado de forma oportuna. Una de las alternativas para diversificar la tan agrarizada economía rural granadina era optar por apoyar a este colectivo, cuyos integrantes la mayor parte del ańo perciben el subsidio agrario, para que encontraran oportunidades de empleo en otros sectores.

Por otra parte, los que eran anteriormente agricultores y ganaderos representan únicamente $8,3 \%$ de los beneficiarios finales encuestados, que se ven obligados a abandonar un sector agrario minifundista sin apenas rentabilidad económica. Este hecho también ha sido registrado en otros estudios para otras regiones rurales de Europa, como el de Shortall (2008) para Irlanda del Norte. Es decir, los PDR no han contribuido de forma significativa a diversificar las actividades, favoreciendo el traspaso de personas del agro hacia otras profesiones, principalmente de servicios. No ha existido la necesaria implicación de los empresarios agrarios, tradicionalmente reacios a realizar nuevas inversiones y a diversificar sus negocios - en definitiva, a modificar sus hábitos y comportamientos laborales-, pero tampoco los GAL han sabido despertar el interés en ellos. Probablemente los agricultores no han apreciado una alternativa válida, una oportunidad de negocio y un apoyo real por parte de los PDR. Aunque tampoco se puede obviar el efecto de descenso de protagonismo de los grupos de presión agrarios en la participación en la toma de decisiones en cuestiones relativas al mundo rural, hecho favorecido también por estas iniciativas, tanto a nivel local como, incluso, a escala comunitaria. Y, por último, no se puede olvidar la poca atención prestada a este colectivo, que se considera prescindible en los clásicos modelos de desarrollo económico.

Las mujeres rurales sufren una doble discriminación, tanto por su género como por su lugar de residencia. De hecho, disponen de menores ofertas laborales allí que en la ciudad e, incluso, en muchas ocasiones son trabajadoras invisibles. "Un

Incluso, indirectamente, los PDR contribuyeron a crear mayor precariedad en el empleo, a causa de la alta temporalidad de los nuevos puestos de trabajo obtenidos en turismo y hostelería y empresas agroalimentarias, situación en que el componente femenino se ha visto especialmente afectado. Además, los empleos que se crearon lo fueron en los municipios más dinámicos, donde las empresas generaban mayor cantidad de puestos de trabajo. Esto también aparece como cuestionable, considerando la primacía dada a los espacios mejor situados, económicamente hablando, en detrimento de aquellos con menores oportunidades laborales y mayor sobredependencia agraria. 
colectivo importante de mujeres que, aunque insertas en la economía productiva, son consideradas estadísticamente como inactivas debido a su inserción no formal" (Camarero \& Oliva, 2004, p. 160). Tampoco se puede soslayar algo muy común en el rural andaluz, como es que en determinadas labores agrarias la mujer está inmersa en la "rueda del temporerismo, trabajo como temporeras durante las campańas agrícolas y percepción posterior del subsidio agrario" (Nuevo, 2000, p. 93). La "huida ilustrada" (Camarero, 2008, p. 73) a la ciudad sigue siendo todavía para muchas de ellas, y más entre las de edad menos avanzada, la única alternativa. De hecho, las oportunidades profesionales en el medio rural son muy reducidas, en especial para las mujeres y más aún para las de mayor formación, muchas de ellas universitarias, ya que las opciones de movilidad, tanto personales como colectivas, son allí más reducidas que en medios urbanos. Ahora bien, de cara a la sostenibilidad social y el desarrollo económico de las áreas rurales, la masculinización es el principal hándicap que puede estar hipotecando el futuro de la vida de los pueblos. En los territorios con PDR de nuestra provincia el número de varones supera al de mujeres en la población activa (165 hombres por 100 mujeres en 2001, según el Censo de Población). Es decir, se perpetúa el solitario “'baile de los solteros' (...), en el que estos herederos se convierten en tristes guardianes del patrimonio familiar, incapaces de conseguir esposas que mantengan y den sostenibilidad social a los mismos" (Bourdieu, 2004, p. 11).

En LEADER I y II, es decir, de 1991 a 1999, no se realizaba ninguna mención especial en los documentos oficiales sobre las desigualdades entre varones y mujeres en el mundo rural. Posteriormente, sí se hace para LEADER +, donde se establecen como prioritarias las estrategias que tengan por objetivo un incremento de empleo y de las actividades destinadas a ellas, concediéndose mejor ponderación y mayores ratios de ayuda para los proyectos impulsados por ellas y/o los que suponen la creación de empleos femeninos, lo que supuso un "contexto favorable para avanzar en la plena integración de las mujeres" (Moyano, 2009, p. 37).

Se puede afirmar con rotundidad que, en gran medida, gracias a los PDR han aparecido emprendedoras en el medio rural, cosa que hace unas décadas era imposible pensar, y que con las nuevas ayudas que tales programas significan se han arriesgado a montar su propia empresa, lo que tiene un elevado efecto demostrativo para las compañeras del mismo género. En otras ocasiones, el logro fue contribuir a sacar de la economía informal a mujeres trabajadoras y empresarias que ya poseían anteriormente un negocio, aunque no declarado. Por tanto, las actuaciones realizadas por emprendedoras presentaron una serie de cualidades positivas intangibles: por su género, constituyeron un factor potenciador del efecto demostrativo de los PDR entre sus paisanas; también lo fueron por desarrollar su propio negocio, su propio proyecto de futuro; y porque en muchas ocasiones ponen en valor determinados recursos endógenos, productos y servicios autóctonos 5 .

Tampoco se debe olvidar que, gracias en parte a los PDR, las mujeres jóvenes están realizando una relectura del medio rural. Gran parte de ellas ha podido elegir ya su lugar, su identidad, accediendo a una oportunidad laboral y de negocio, e incluso a una participación real en las tareas de poder de estas comarcas, siendo modernas y de pueblo al mismo tiempo. 
No obstante lo anterior, a pesar de que las mujeres se están beneficiando de las actuaciones, lo hacen "aún en bastante menor medida que los hombres" (Calatrava, 2002, p. 75) (Cuadro 3). Sabaté (2009) expresa rotundamente que "las políticas de desarrollo rural han incorporado de forma insuficiente la dimensión de género" (p. 103). Incluso, determinados yacimientos de empleo, como turismo, transformación artesanal de productos agroalimentarios, o cuidado de ancianos, promovidos por los PDR, "se relacionan con actividades tradicionalmente asumidas por las mujeres en el ámbito doméstico. Siendo una oportunidad de profesionalización para las mujeres aunque presentan también un riesgo de reproducción de los roles de género tradicionales" (Sampedro, 2008, p. 91). De hecho, para la provincia, el número de empresarias beneficiadas por los PDR fue muy reducido (Cuadro 3): tan solo 31,8\% (140) respecto del total de emprendedores autónomos (440), y eso sin tener presente otro tipo de sociedades mercantiles, donde, sin duda, la representación femenina fue incluso inferior. Esta cifra se encuentra ligeramente por debajo de la realizada por LEADER il para el caso de Espańa, que fue de 35\%, aproximadamente (Martínez, 2004). Entre las empresarias en referencia encontramos dos perfiles diferentes: por una parte, mujeres jóvenes formadas; y por otra, mujeres de mayor edad sin formación, que han superado ya la etapa de crianza de los hijos.

Ahora bien, la escasez de mujeres entre las emprendedoras acogidas se fue paliando según se avanzaba en el tiempo, de 22\% en el periodo 1991-1993, a un meritorio 38,2\% en 2000-2006. Aun así, este último periodo dista mucho de los resultados de LEADER + para Cataluña, donde "las mujeres fueron responsables de algo más de la mitad de los proyectos promovidos individualmente" (Viladomiu, Rosell \& Frances, 2010, p. 181). Asimismo, las mujeres emprendedoras son, por regla general, más jóvenes que los emprendedores del sexo contrario (Gráfico 1): mientras que $72,7 \%$ de las empresarias posee menos de 40 años, en los hombres solo el $44 \%$ posee menos de esa edad.

Las mujeres fueron pioneras en la implantación de casas y hoteles rurales, y ello a pesar de que encontraron serias dificultades de financiación y apoyo familiar. Ahora bien, generalmente las involucradas no eran esposas de agricultores, por lo que el turismo no funcionó como una actividad complementaria de la explotación familiar. Además, y como se ha demostrado en similares estudios (Sparrer, 2003), en gran parte de los casos se trataba de "empresarias ficticias", que aparecían como emprendedoras por razones fiscales; es decir, que aunque la mujer figuraba como propietaria, no desempeñaba las funciones de gerente, quedando su función relegada a las labores domésticas tradicionales: preparar las comidas y cenas, limpieza, lavar y planchar, etcétera.

Por tanto, hay que apostar por iniciativas donde la mujer tenga una mayor representación. Como apuntan Viladomiu et al. (2010), "nuevas empresas, pequeñas iniciativas, sector servicios y muy especialmente servicios personales, comercios, etc." (p. 204). La plena participación de las mujeres en el desarrollo rural puede contribuir a paliar tres problemas básicos del medio rural granadino: su elevado desempleo; la diversificación de la economía, donde ellas juegan un papel primordial en sectores como servicios de proximidad y turismo; así como su imprescindible participación a la hora de invertir las tendencias demográficas de estas comarcas. 
CUADro 3 Emprendedores individuales beneficiados por Programas de Desarrollo Rural (PDR) en la provincia de Granada, según sexo, por periodos

\begin{tabular}{|l|c|c|c|c|c|c|c|c|}
\cline { 2 - 9 } \multicolumn{1}{c|}{} & $\begin{array}{c}\text { I99I- } \\
\text { I993 } \\
\text { ABS. }\end{array}$ & $\begin{array}{c}\text { I99I- } \\
\text { I993 } \\
\text { REL. }\end{array}$ & $\begin{array}{c}\text { I995- } \\
\text { I999 } \\
\text { ABS. }\end{array}$ & $\begin{array}{c}\text { I995- } \\
\text { I999 } \\
\text { REL. }\end{array}$ & $\begin{array}{c}\mathbf{2 0 0 0 -} \\
\mathbf{2 0 0 6} \\
\text { ABS. }\end{array}$ & $\begin{array}{c}\mathbf{2 0 0 0 -} \\
\mathbf{2 0 0 6} \\
\text { REL. }\end{array}$ & $\begin{array}{c}\text { TOTAL } \\
\text { ABS. }\end{array}$ & $\begin{array}{c}\text { TOTAL } \\
\text { REL. }\end{array}$ \\
\hline Mujer & 9 & 22,0 & 55 & 27,5 & 76 & 38,2 & 140 & 31,8 \\
\hline Hombre & 32 & 78,0 & 145 & 72,5 & 123 & 61,8 & 300 & 68,2 \\
\hline Total & 41 & 100,0 & 200 & 100,0 & 199 & 100,0 & 440 & 100,0 \\
\hline
\end{tabular}

FUENTE INFORMES FINALES DE LOS GAL DE LA PROVINCIA DE GRANADA (I992-2006)

Por otra parte, retener a los jóvenes en el medio rural se configura como uno de los principales retos que se plantean en estos momentos los PDR. Pero contrariamente al caso anterior, existió poca presencia relativa de emprendedores beneficiados de menos de 35 años: 22,6\% ${ }^{6}$. En cierta medida, los GAL, al valorar la experiencia y la solvencia económica en la selección de proyectos, han limitado considerablemente el acceso a las ayudas a gran cantidad de jóvenes emprendedores. Además, "la falta de confianza de la familia para ceder recursos, la falta de recursos propios y el interés en un primer momento por un empleo asalariado" (Langreo, 2000, p. 79) también han contribuido a la poca presencia de emprendedores en edad temprana. Aun así, a pesar de su escaso número, las iniciativas de estos empresarios de corta edad tuvieron un elevado efecto imitación a causa de su elevado componente innovador, ya que tendieron en mayor medida a crear empresas, además de apostar por nuevos yacimientos de empleo en el sector servicios que exigían una elevada formación y cualificación. "Atrevidos, imprudentes en algunos casos, se trata de empresarios que han tenido que flexibilizar negocios familiares, hipotecar su propio patrimonio (...), afrontar nuevas relaciones con clientes y proveedores" (Villena, 2000, p. 71).

Tampoco ha sido significativa la participación de "nuevos" habitantes rurales en los PDR. De hecho, si los identificamos como aquellos de fuera de la provincia, apenas alcanzaron $9,8 \%$; y dentro de estos, únicamente $2 \%$ se debe a emprendedores de otro Estado; 3,3\% proviene de otra región, y 4,7\% de otra provincia. Su presencia podría considerarse representativa en las Alpujarras (25\%), confirmando aquello de que el fenómeno de la contraurbanización no es universal, no incide por igual en todos los territorios rurales e, incluso, que sus implicados poseen diferentes perfiles. Si consideramos el razonamiento de Bosworth y Willett (2011), según quienes "los que experimentan una vuelta al campo, y echan raíces en la comunidad local son los mejor situados para introducir nuevas formas de capital humano y social y suministrar conexiones ventajosas al área local” (pp. 209-210), sería conveniente una mayor implicación de esos grupos en los programas.

Por su parte, comerciantes ${ }^{7}$, empresarios de la construcción (tan solo 1,8\% de los beneficiarios finales), o funcionarios $(2,8 \%)$ que viven en el territorio rural, a pesar

6 Información extraída de las entrevistas a los emprendedores.

7 Este empresariado, clásico en el medio rural, continúa una arraigada tradición familiar, controlando el comercio local y la prestación de determinados servicios, aunque con la única intención de mantener su posición de pequeńa burguesía y mantener el statu quo, con una clara actitud conservadora. 
de disponer de solvencia económica para invertir e implicarse en los PDR, en la mayor parte de las ocasiones no han respondido de manera conveniente. Ello se debe probablemente a sus pocas expectativas frente a la escasez de los fondos, a los mayores beneficios que podían encontrar en otros ámbitos -es el caso de la construcción-, y a una bastante generalizada actitud conservadora.

\section{¿Quiénes sí?}

En el extremo opuesto se ha apreciado que los beneficiados en mayor medida han sido empresarios autónomos y de pymes, todos ellos con una larga trayectoria empresarial, ya consolidados, autoempleados; y en menor medida, trabajadores con una larga trayectoria profesional. Su experiencia anterior a la inversión subvencionada por los PDR está relacionada con la hostelería y el turismo (19,6\%), pequeñas empresas de servicios (16,6\%) y agroalimentarias (16,6\%). En menor medida, han surgido nuevos emprendedores jóvenes sin trayectoria empresarial anterior (15,7\%), aunque con un elevado nivel formativo y de cualificación, y otros emprendedores de mayor edad que proceden de actividades artesanales (13,6\%).

Por otra parte, el tamaño de las empresas beneficiadas, en lo que a número de trabajadores respecta, es limitado. De hecho, predominan el autoempleo y las microempresas (menos de 10 trabajadores), englobando ambas el 73\%, y siendo minoritarias las que poseen más trabajadores (27\%). Este hecho, negativo a la hora de competir en costes y acrecentar el problema del minifundismo empresarial del espacio rural granadino, es positivo, y por diversas razones: estas microempresas presentan mayor flexibilidad y adaptación a los cambios del mercado; pueden apostar por la diferenciación y calidad del producto; poseen una relación más cercana y atención personal con el cliente; existe en ellas un mayor contacto y complicidad entre empresario y trabajadores; y, por último, al ser empresas endógenas, con empresarios locales que emplean recursos propios, establecen una mayor ligazón con el territorio, lo que las fija en un determinado municipio, dificultando su "deslocalización" o su cierre ante las cíclicas crisis económicas.

En cuanto a la población empresaria, el $46,1 \%$ se ubica en el intervalo de edad de 35-44 años. Es decir, la mayor parte de los emprendedores acogidos ha invertido cuando se ha encontrado en una fase intermedia de su vida empresarial, con la trayectoria y madurez suficientes para acometer ampliaciones o modernizaciones de su negocio. Lo anterior se corrobora con el hecho de que los proyectos acometidos para generar nuevos negocios han sido los minoritarios $(15,7 \%)$, predominando los de ampliación o modernización (84,3\%). Es decir, lo más frecuente ha sido llevar a cabo la inversión en una edad adulta-joven.

Por tanto, la ayuda ha llegado en mayor medida a los grupos políticamente más influyentes y económicamente más pudientes. La mayor parte $(78 \%$, según datos de la encuesta), microempresarios varones ya consolidados y de edad adulta-joven (54\%, hombres de entre 35 y 54 ańos). 
GRÁfico 1 | Sexo y edad de los emprendedores beneficiados por Programas de Desarrollo Rural (PDR) en la provincia de Granada (1991-2006)

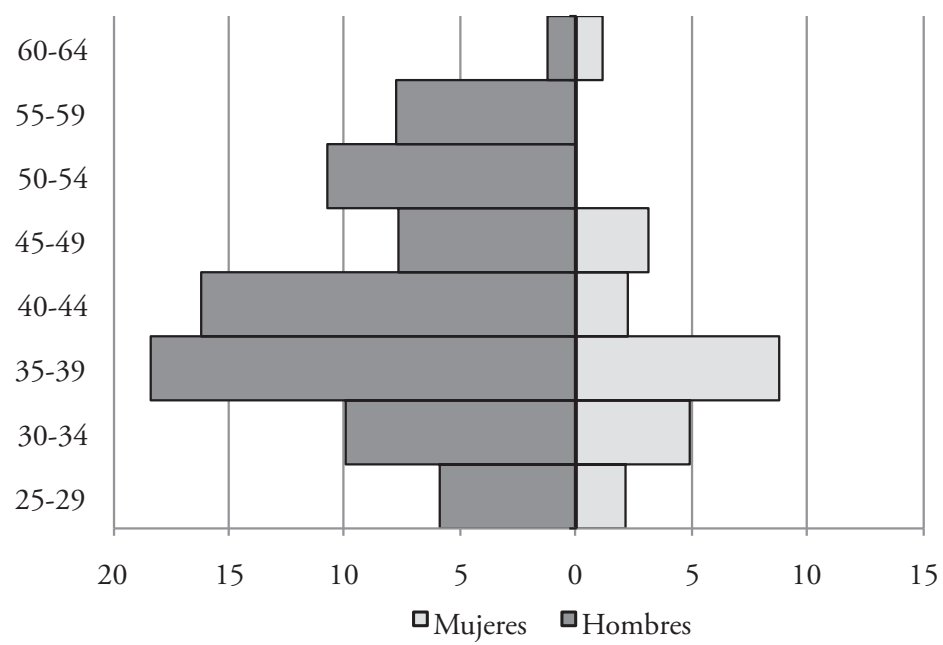

FUENTE ELABORACIÓN PROPIA. ENCUESTA REALIZADA A LOS EMPRENDEDORES BENEFICIADOS POR PDR

\section{¿Dónde se localizaron?}

Los PDR refuerzan los diferentes roles desempeñados por cada territorio en su inserción en el proceso económico global, favoreciendo aquellos espacios con empresarios solventes y dinámicos, las ciudades medias o centros comarcales -agrociudades, para el caso andaluz-, como lugares suministradores de bienes y servicios de función superior, con mayores economías de escala, y donde se concentra la recuperación demográfica. Se han visto penalizados los marginados con tendencia a la decadencia social y económica y al despoblamiento (Cejudo \& Navarro, 2003; 2009; 2012) (Figura 2).

FIGURA 2 Número de emprendedores privados para la provincia de Granada (1991-2006)

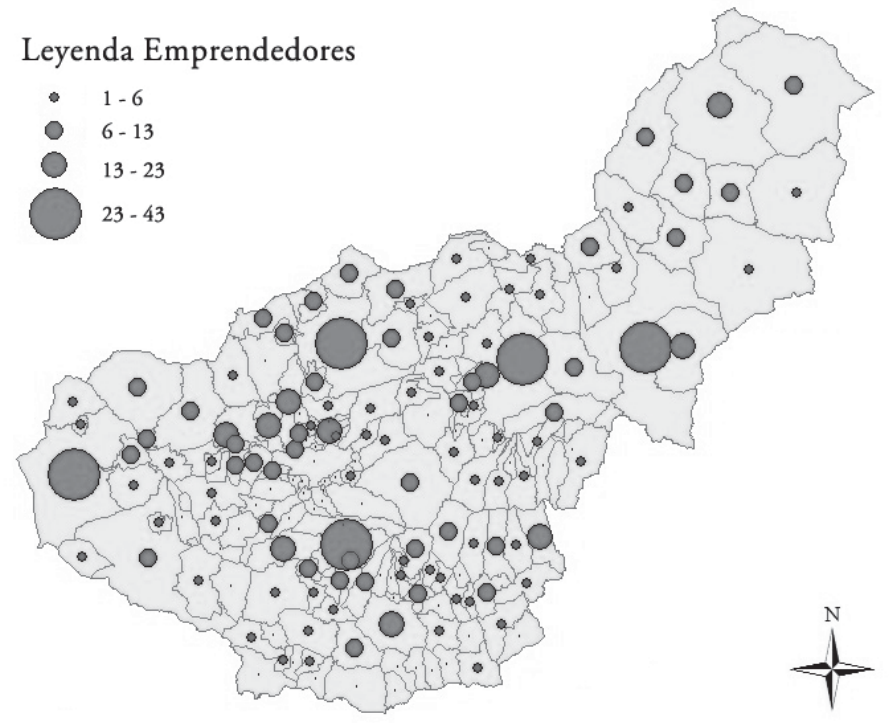

FUENTE INFORMES FINALES DE LOS GAL 


\section{¿Cuánto invirtieron?}

Cuestión primordial esta, ya que nos permite, por un lado, determinar la incidencia que pueda tener ese montante económico global en el territorio; y, por otro, estudiar la implicación de la población local, al menos en lo que respecta a su participación económica. En lo que se refiere al primer aspecto, la incidencia que pueda tener esa inversión en el territorio rural provincial, a causa de su reducida cuantía -unos 201 millones de euros en total- para el espacio provincial y para quince ańos de aplicación, resulta claramente insuficiente, si se atiende a la cuantía de otras políticas - como, por ejemplo, la Política Agraria Común (PAC) clásica y considerando los cambios en el tejido productivo que ha podido generar. Así lo afirman también otros autores para otros territorios, como Nardone, Sisto y Lopolito (2010), al señalar que "la Iniciativa Leader es una política a pequeña escala que opera en territorios rurales con un modesto montante de recursos financieros” (p. 64). Más aún si se tiene en cuenta que la inversión media por habitante ha sido ínfima, de 498 euros, y que la aportación privada movilizada no ha alcanzado los 260 euros. Si acaso el efecto demostrativo entre los empresarios, en particular, y en la población rural, en general, puede haber sido el mayor aliciente e impacto de los PDR.

En lo que respecta a la cuantía económica movilizada en cada uno de los proyectos, se puede señalar que también es modesta (Cuadro 4). En cada uno de ellos se invierte unos 140.000 de media, de los que el $72 \%$ es aportado por el beneficiario (102.000 euros). Incluso la inversión es 40\% inferior en autónomos, el colectivo con menor capacidad de inversión (86.000), entre los que la movilización particular de dinero apenas alcanza los 61.000 euros. Es oportuno recordar que las poblaciones de los territorios rurales poseen menor capacidad económica y, por tanto, inversora, y que esta se encuentra muy restringida a los grupos y colectivos que forman las elites locales que venimos señalando. Por otra parte, la cantidad que se invierte, contrariamente a lo que se podría pensar, es menor si se aborda la creación de una empresa que si se amplía o moderniza una ya existente -132.000 euros de media para la primera y 145.000 para la segunda-, aunque la subvención pública, por supuesto, es mayor para crear empresas (29,7\% frente al $27,5 \%)$. Todo indica que los empresarios ya consolidados poseen mayor solvencia económica que los nuevos y jóvenes emprendedores, lo que les permite realizar una mayor inversión con un menor riesgo.

También son inferiores las cantidades invertidas por mujeres emprendedoras (104.000 euros). Este hecho nos indica que gran parte de los negocios en los que ellas emprenden van a funcionar como complemento al ingreso principal de la familia, proporcionado por el hombre. Para ellas, "la motivación principal fue la de complementar los ingresos del hogar” (Pastor \& Esparcia, 1998, p. 540). Ahora bien, las que son al mismo tiempo mujeres y jóvenes asumen un riesgo empresarial importante (154.000 euros), que, cuestionablemente, no se acompańa con una subvención pública acorde (24\%). De ahí que la mayoría de ellas considere las cifras como "escasas", "discrecionales" o "circunstanciales" (Cruces \& Palenzuela, 2006, p. 303).

Sobre la denominada PAC clásica, puede verse Compés, López y Martínez (2011). 
Si son los colectivos de mujeres y jóvenes tan necesarios e importantes para el futuro de los territorios rurales, ¿por qué sus inversiones son minusvaloradas en subvención pública?, ¿por qué el colectivo de hombres adultos cuenta con un promedio de $4 \%$ más de ayuda pública? Incluso algo similar, aunque con menos diferencia, ocurre en el colectivo de jóvenes, y en el de mujeres respecto de aquel, y en el de autónomas, autónomos jóvenes y autónomas jóvenes, respecto al del total de autónomos. Por tanto, además de la carencia de capital y patrimonio propio y de la escasa existencia de respaldo financiero, el apoyo público, en realidad, no alcanza la importancia que se proclama para el conjunto del periodo considerado. Afortunadamente, a partir del 2000, y gracias al respaldo institucional promovido para LEADER +, ya anteriormente seńalado, se asiste a un cambio de rumbo encaminado a un mayor apoyo económico público a estos colectivos.

Además, y aunque como se ha señalado anteriormente los porcentajes de subvención pública son significativos y a "fondo perdido" -oscilando entre el $24 \%$ y el $30 \%-$, la mayor parte de los emprendedores $(79,4 \%)$ comenta que hubieran llevado a cabo la inversión pese a no haber recibido la ayuda. El efecto despegue y motivador no ha sido importante, ya que eran inversiones previstas por parte de los empresarios, dirigidas fundamentalmente a modernizar o a ampliar los negocios ya existentes $(72,9 \%)$, y no a crearlos $(27,1 \%)$, funcionando, por tanto, la contribución económica de estos PDR como algo secundario.

CUADRo 4 Inversión media según tipo de empresa y atendiendo a creación/ consolidación en los Programas de Desarrollo Rural (PDR) de la provincia de Granada (1991-2006)

\begin{tabular}{|l|r|r|r|c|c|}
\cline { 2 - 6 } \multicolumn{1}{c|}{} & TOTAL & PRIVADO & PÚBLICO & $\begin{array}{c}\% \\
\text { PRIVADO }\end{array}$ & $\begin{array}{c}\% \\
\text { PÚBLICO }\end{array}$ \\
\hline Media de inversión por proyecto & 142.752 & 102.984 & 39.768 & 72,1 & 27,9 \\
\hline Autónomos & 86.141 & 61.256 & 24.885 & 71,1 & 28,9 \\
\hline Autónomos jóvenes & 93.641 & 69.136 & 24.505 & 73,8 & 26,2 \\
\hline Autónomas mujeres & 86.547 & 61.663 & 24.884 & 71,2 & 28,8 \\
\hline Autónomas mujeres-jóvenes & 93.675 & 69.785 & 23.890 & 74,5 & 25,5 \\
\hline Creación de empresa & 131.860 & 92.731 & 39.129 & 70,3 & 29,7 \\
\hline Consolidación de empresa & 145.119 & 105.246 & 39.873 & 72,5 & 27,5 \\
\hline Dirigidas por jóvenes $\left(^{*}\right)(* *)$ & 154.815 & 113.423 & 41.392 & 73,3 & 26,7 \\
\hline Dirigidas por mujeres $\left(^{* *}\right)$ & 104.558 & 75.867 & 28.691 & 72,6 & 27,4 \\
\hline Dirigidas por mujeres jóvenes $(* *)$ & 154.255 & 117.253 & 37.002 & 76 & 24 \\
\hline Dirigidas por hombres adultos & 150.349 & 108.399 & 41.950 & 72,1 & 27,9 \\
\hline
\end{tabular}

* Se consideran jóvenes los emprendedores que poseen 35 ańos y menos.

** Atendiendo a la estadística proporcionada por los GAL, cuando más del $25 \%$ de socios de una empresa posee 35 y menos ańos y/o son mujeres, se la incluye como empresa dirigida por jóvenes y/o mujeres.

FUENTE INFORMES FINALES DE LOS GAL DE LA PROVINCIA DE GRANADA (I99I-2006) 
Se asiste al hecho, entonces, de que las cantidades totales invertidas en el medio rural provincial son ridículas, e incapaces de originar cambios estructurales y significativos en el tejido productivo. Además, los proyectos en referencia, aunque aumentan la cantidad de empresas y generan un elevado efecto demostrativo, no consiguen paliar la apatía empresarial y la pasividad de las clases solventes. Pero, además, las cuantías arriesgadas por colectivos deprimidos -como son emprendedores jóvenes y de sexo femenino-, aunque esenciales para el futuro de estos territorios, no se acompañan de los oportunos montantes de ayudas públicas, e incluso son penalizados respecto de los percibidos por hombres adultos.

\section{Principales causas de las ausencias y presencias}

Las razones que explican la participación o la exclusión de determinados colectivos como beneficiarios finales de los PDR y, en definitiva, en el poder local rural mediante la política de desarrollo rural, son múltiples y complejas, internas y externas.

El dominio excesivo de los GaL y de la toma de decisiones por parte de las autoridades locales, ya señalada también por otros autores (Buller, 2000, Esparcia et al., 2000), ha generado un partenariado ficticio, estando sobrerrepresentado el papel de las administraciones públicas. Los alcaldes y representantes políticos, tanto locales como regionales, han controlado las Juntas Directivas, lo que ha implicado que utilicen los PDR para fomentar el clientelismo. Tales dirigentes políticos han decidido, por tanto, los objetivos, los proyectos que serán subvencionados y los grupos beneficiarios. Como se ha demostrado en otros casos, "las autoridades locales utilizaron LEADER como moneda de cambio para asegurar su reelección (...) en detrimento de la promoción de la participación comunitaria y la inclusión social" (Furmankiewicz, Thompson \& Zielińska, 2010, p. 53).

También ha ocurrido que una serie de grupos de presión (sectoriales, territoriales, económicos y políticos) han controlado los PDR y el poder local, monopolizando el control de los recursos y limitando la participación de colectivos desfavorecidos del medio rural y los beneficios destinados a ellos (Esparcia et al., 2000; Van der Ploeg et al., 2000).

Otro motivo ha sido que los técnicos, tanto del propio GaL como de otras administraciones locales y regionales, han reforzado el control descendente. Ha existido una alta contradicción entre el enfoque ascendente que propugnan los PDR y el tradicional dirigista de la administración. La administración ha sido reticente a compartir el poder. Incluso, en gran parte de las ocasiones, los GAL no decidían sobre los proyectos y fondos. Esto ha conllevado que los PDR sean entendidos como programas externos y no como instrumentos del desarrollo endógeno.

Por otra parte, se priorizó lo económico, a los empresarios y los resultados finales, y no los procesos. Un excesivo énfasis en favorecer fondos para proyectos individuales de promotores, la obligatoriedad de adelantar con fondos propios la inversión, la lentitud en otorgarlos y la elevada complejidad del proceso, tampoco han favorecido la extensión de las iniciativas a otros colectivos.

Además, las actividades en formación no se han adaptado en suficiente proporción a las necesidades y carencias laborales de los colectivos marginales. La mejora 
de sus habilidades profesionales hubiera redundado en una mayor participación en el mercado de trabajo y una mayor empleabilidad, generando así mayor riqueza y productividad, menores probabilidades de desempleo y mayores de autorrealización personal y calidad de vida.

Y por último, obviamente, tampoco puede olvidarse señalar la propia desestructuración de la sociedad rural, con una muy escasa organización y capacitación de amplios colectivos sociales, especialmente los marginales: baja formación y cualificación, predominio de la cultura del subsidio, débil tradición de implicación en el gobierno local, "falta de cultura democrática y de participación" (Esparcia et al., 2000, p. 95), entre otros.

En definitiva, la participación de los colectivos marginales ha sido reducida por la concepción de los PDR como instrumento de poder.

\section{Conclusiones}

Entre los beneficiarios finales de los PDR predominaron empresarios de edad adultajoven con un negocio ya consolidado y -cómo no- cercanos al poder político y económico; varios colectivos se vieron mayormente excluidos y apenas pudieron implicarse en el desarrollo de sus territorios: mujeres, jóvenes, trabajadores del campo y desempleados, entre otros.

Es decir, el grado de participación de unos colectivos y otros no fue similar. Aparecen, así, grupos claramente definidos: los marginados, que pierden protagonismo, influencia y poder (como empresarios agrarios y jornaleros del campo); los participantes efectivos (empresarios y profesionales ligados a las elites políticas, económicas y sociales); $y$, por último, los que aspiran a integrarse en mayor medida en los PDR (neorrurales, desempleados, jóvenes, mujeres, entre otros).

La instrumentalización de los GAL por el poder político, social y económico ha hecho que los PDR refuercen las estructuras de poder y, al mismo tiempo, contribuyan a la exclusión de los grupos con menores posibilidades. La actual postura de la Comisión Europea hacia la participación popular en el desarrollo rural no es más que mera retórica. En la práctica, la filosofía y el método LEADER no encajan en la búsqueda de la equidad socioterritorial, dominados como están por un marcado carácter neoliberal. El desarrollo rural solo tiene el potencial de desafiar los procesos de exclusión social y territorial si se permite incluir a los sin poder.

Aunque es la globalización económica la que marca las pautas en las micropolíticas locales y la que contribuye a esa polarización social, los agentes de desarrollo rural, junto con una más amplia participación de la población local, pueden reconducir, intervenir y tener influencia en la particular inserción en ella de cada territorio. 


\section{Referencias bibliográficas}

Böcher, M. (2008). Regional governance and rural development in Germany: the implementation of Leader +. Sociologia Ruralis, 48(4), 372-388. doi:10.1111/j.1467-9523.2008.00468.x

Bosworh, G. \& Willett, J. (2011). Embeddedness or escapism? Rural perceptions and economic development in Cornwall and Northumberland. Sociologia Ruralis, 51(1), 195-214. doi: 10.1111/j.1467-9523.2011.00533.x

Bourdieu, P. (2004). El baile de los solteros. Madrid: Anagrama.

Buller, H. (2000). Recreating rural territories: LEADER in France. Sociologia Ruralis, 40(2), 190199. doi: $10.1111 / 1467-9523.00141$

Calatrava, J. (2002). Mujer y desarrollo rural en la globalización: de los proyectos asistenciales a la planificación de género. Información Comercial Española, 803, 73-90. Información Comercial Española, ICE: Revista De Economía, 803, 73-90. En http://dialnet.unirioja.es/ servlet/articulo?codigo $=292785$

Camarero, L. (2008). ¿Por qué se van las mujeres? El continuum de movilidad como hipótesis explicativa de la masculinización rural. Revista Española de Investigaciones Sociológicas, 124, 73-106. En http://dialnet.unirioja.es/servlet/articulo?codigo=2712844

Camarero, L. \& Oliva, J. (2004). Las trabajadoras invisibles de las áreas rurales: un ejercicio estadístico de estimación. Empiria, Revista de Metodología de Ciencias Sociales, 7, 159179. En http://dialnet.unirioja.es/servlet/articulo? codigo $=1374492$

Cejudo, E. \& Navarro, F. (2003). El reparto territorial de los programas de desarrollo rural. El caso de la provincia de Granada. Anales de Geografía de la Universidad Complutense, 23, 131162. En http://revistas.ucm.es/index.php/AGUC/article/view/AGUC0303110131A

Cejudo, E. \& Navarro, F. (2009). La inversión en los programas de desarrollo rural. Su reparto territorial en la provincia de Granada. Anales de Geografia de la Universidad Complutense, Vol. 29, 2, 37-64.

Cejudo, E. \& Navarro, F. (2011). Quince años de aplicación de los programas de desarrollo rural. Desigualdades sociales y territoriales en la provincia de Granada. Scripta Nova, 16(390), 1-32. En http://www.ub.edu/geocrit/sn/sn-390.htm

Comisión Europea (2009). Sexto informe sobre la cohesión económica y social, COM (2009) 295 final. Bruselas. Oficina de Publicaciones Oficiales de las Comunidades Europeas.

Compés, R., López, E. \& Martínez, V. (2011). Evolución de la PAC y evaluación de las propuestas legislativas para la reforma de 2013. En J. M. García \& F. Sineiro (Coords.), Apoyo público a la agricultura española (pp. 25-69). Madrid: Ministerio de Medio Ambiente y Medio Rural y Marino, España. En http://goo.gl/OMM67h

Cruces, C. \& Palenzuela, P. (2006). Emprendedoras rurales en Andalucía. Posibilidades y límites de sus estrategias. Revista de Estudios Agrosociales y Pesqueros, 211, 239-305. En http:// dialnet.unirioja.es/servlet/articulo?codigo $=2307931$

Esparcia, J. (2011). Los grupos de acción local en el Programa leader en España. En Ministerio de Medio Ambiente y Medio Rural y Marino (MARM), LEADER en España (1991-2011). Una contribución activa al desarrollo rural (pp. 111-139). Madrid. MARM. En http://goo. $\mathrm{gl} / \mathrm{LmJXdX}$

Esparcia, J., Noguera, J. \& Pitarch, M. (2000). Leader en España: desarrollo rural, poder, legitimación, aprendizaje y nuevas estructuras. Documentos de Análisis Geográfico, 37, 95-113. En http://ddd.uab.es/pub/dag/02121573n37p95.pdf 
Farinós, J. (2008). Gobernanza territorial para el desarrollo sostenible: estado de la cuestión y agenda. Boletín de la Asociación de Geógrafos Españoles, 46, 8-32. En http://www.fiaes.org. sv/main.php?lng=0\&id=92\&s=7

Furmankiewicz, M., Thompson, N. \& Zielińska, M. (2010). Area-based partnerships in rural Poland: The post-accession experience. Journal of Rural Studies, 26(1), 52-62. http:// dx.doi.org/10.1016/j.jrurstud.2009.05.001

Jorquera, D. (2011). Gobernanza para el desarrollo local. Documento de Trabajo no 6. Proyecto Conocimiento y Cambio en Pobreza Rural y Desarrollo. Rimisp, Centro Latinoamericano para el Desarrollo Rural. Santiago, Chile. En http://goo.gl/aAv5Hy

Junta de Andalucía (2008). Programa de Desarrollo Rural de Andalucía 2007-2013. Sevilla: Comité de Desarrollo Rural. Junta de Andalucía. En http://goo.gl/BQGx0L

Kearney, B., Boyle, G. \& Walsh, J. (1995). EU LEADER I Initiative in Ireland. Evaluation and recommendations. Dublin: Department of Agriculture, Food and Forestry.

Lacambra, V. (2001). Desarrollo rural en los espacios rurales europeos. Elementos de desigualdad territorial. Revista Catalana de Sociología, 14, 253-276. En http://www.raco.cat/index. $\mathrm{php} /$ RevistaSociologia/article/view/222340

Langreo, A. (2000). Innovaciones y desarrollo rural: nuevas iniciativas de empleo y juventud. Estudios de Juventud, 48, 73-81. En http://www.injuve.es/sites/default/files/2012/44/ publicaciones/revista-48-capitulo7.pdf

Martínez, E. (Dir. Equipo investigación: López, N., Martín, F. Sabaté, A. \& Sáez, E.). (2004). Mujeres emprendedoras en el desarrollo rural. Atlas Regional de España de la participación de las mujeres rurales emprendedoras en la Iniciativa Comunitaria LEADER II. Madrid: Ministerio de Trabajo y Asuntos Sociales. En http://goo.gl/JKzsZh

Midmore, P. (1998). Rural policy reform and local development programmes: Appropriate evaluation procedures. Journal of Agricultural Economics, 49(3), 409-426. doi: 10.1111/ j.1477-9552.1998.tb01281.x

Moscoso, D. (2005). Las dimensiones del desarrollo rural y su engranaje en los procesos de desarrollo comarcal de Andalucía. Revista de Estudios Regionales, 73, 79-104. En http:// www.revistaestudiosregionales.com/pdfs/pdf817.pdf

Moyano, E. (2009). Las mujeres del medio rural. De la invisibilidad al reconocimiento social. En Unión de Pequeños Agricultores (UPA)(Ed.), Agricultura familiar en España 2009 (pp. 3439). Madrid: UPA. En http://www.upa.es/anuario_2009/pag_034-039_eduardomoyano. pdf

Nardone, G., Sisto, R. \& Lopolito, A. (2010). Social capital in the LEADER Initiative: a methodological approach. Journal of Rural Studies, 26(1), 63-72. http://dx.doi. org/10.1016/j.jrurstud.2009.09.001

Nuevo, T. (2000). Las mujeres jóvenes en el medio rural; clave para el desarrollo. Estudios de Juventud, 48, 91-96.

Pastor, C. \& Esparcia, J. (1998). Alternativas económicas en el ámbito rural interior. El papel de las mujeres en el desarrollo rural. Cuadernos de Geografia, 64, 527-542. En http://roderic. uv.es/handle/10550/31026

Ramos, E. \& Romero, J. (1995). Del "productivismo" al "ruralismo": una reflexión sobre la política agraria en Andalucía. Revista de Estudios Agrosociales, 169, 175-212. En http:// www.magrama.gob.es/ministerio/pags/Biblioteca/Revistas/pdf_reas\%2Fr169_06.pdf 
Ray, C. (2001). Transnational cooperation between rural areas: elements of a political economy of EU rural development. Sociologia Ruralis, 41(3), 279-295. doi: 10.1111/14679523.00183

Rodríguez, F. (2000). Desarrollo rural en las montañas andaluzas. Un análisis desde la sostenibilidad. Cuadernos Geográficos de la Universidad de Granada, 30, 97-121.

Romero, J. \& Farinós, J. (2011). Redescubriendo la gobernanza más allá del buen gobierno. Democracia como base, desarrollo territorial como resultado. Boletín de la Asociación de Geógrafos Españoles, 56, 295-319. En dialnet.unirioja.es/descarga/articulo/3722490/1. pdf

Sabaté, A. (2009). La dimensión de género en las políticas españolas y europeas de desarrollo rural. En Unión de Pequeños Agricultores (UPA) (Ed.), Agricultura familiar en España (pp. 103108). Madrid: UPA. En www.upa.es/anuario_2009/pag_103-108_anasabate.pdf

Sampedro, R. (2008). Conciliación de la vida familiar y laboral en el medio rural: género, trabajo invisible e "idilio rural". En V. Frades (Ed.), Mujeres rurales. Estudios multidisciplinares de género (pp. 81-93). Salamanca: Ediciones Universidad de Salamanca.

Scott, M. (2004). Building institutional capacity in rural Northern Ireland: the role of partnership governance in the LEADER II programme. Journal of Rural Studies, 20(1), 49-59. http:// dx.doi.org/10.1016/S0743-0167(03)00042-1

Shortall, S. (2008). Are rural development programmes socially inclusive? Social inclusion, civic engagement, participation, and social capital: exploring the differences. Journal of Rural Studies, 24(4) 450-457. http://dx.doi.org/10.1016/j.jrurstud.2008.01.001

Shucksmith, M. (2010). Disintegrated rural development? Neo-endogenous rural development, planning and place-shaping in diffuse power contexts. Sociologia Ruralis, 50(1), 1-14. doi: $10.1111 /$ j.1467-9523.2009.00497.x

Sparrer, M. (2003). Género y turismo rural. El ejemplo de la costa coruñesa. Cuadernos de Turismo, 11, 181-197. En http://revistas.um.es/turismo/article/view/19441

Stoker, G. (1998). El buen gobierno como teoría: cinco propuestas. Revista Internacional de Ciencias Sociales, 155. En http://www.unesco.org/issj/rics155/titlepage155.html.

Tovey, H. (2008). Rural sustainable development in the knowledge society era. Sociologia Ruralis, 48(3), 185-199. DOI: 10.1111/j.1467-9523.2008.00460.x

Van der Ploeg, J. D., Renting, H., Brunori, G., Knickel, K., Mannion, J., Marsden, T., ... Ventura, F. (2000). Rural development: from practices and policies towards theory. Sociologia Ruralis, 40(4), 391-408. doi: 10.1111/1467-9523.00156

Viladomiu, L., Rosell, J. \& Frances, C. (2010). Factores determinantes de la participación de la mujer como promotoras de proyectos Leader +. Revista Española de Estudios Agrosociales y Pesqueros, 226, 177-206. En http://goo.gl/IvAbqn

Villena, J. (2000). El joven empresario rural. Estudios de Juventud, 48, 69-71. En http://www. injuve.es/sites/default/files/2012/44/publicaciones/revista-48-capitulo6.pdf

Ward, N. \& McNicholas, K. (1998). Reconfiguring rural development in the uK: objective 5b and the new rural governance. Journal of Rural Studies, 14(1), 27-39. http://dx.doi. org/10.1016/S0743-0167(97)00045-4

Woods, M., Edwards, B., Anderson, J. \& Gardner, G. (2007). Leadership in place: elites, authority and agency in British rural community governance. En L. Cheshire, V. Higgins \& E. Lawrence (Eds.), Rural governance: international perspectives (pp. 75-91). Londres: Routledge. 
